\title{
Sentidos y significados del trabajo: un análisis con base en diferentes perspectivas teórico-epistemológicas en Psicología*
}

Senses and Meanings of Work: an Analysis Based on Different Theoretical-Epistemological Perspectives in Psychology

\author{
SUZANA DA ROSA TOLFO** \\ Maria Chalfin Coutinho \\ DAVI BAASCH \\ JoANA SOARES CUGNIER \\ Universidade Federal de Santa Catarina, \\ Florianópolis, Brasil
}

Para citar este artículo.Da Rosa, S., Chalfin, M., Baasch, D., \& Soares, J. (2011). Sentidos y significados del trabajo: un análisis con base en diferentes perspectivas teórico-epistemológicas en Psicología. Universitas Psychologica, 10(1), 175-188.

* Naturaleza del artículo: ensayo.

** Departamento de Psicologia/CFH-UFSC-Campus Universitário - Trindade - Florianópolis, SC CEP: 88040-970.E-mails: srtolfo@yahoo.com.br ; chalfin@mbox1.ufsc.br; savibaasch@hotmail.com; jocugnier@yahoo.com.br

RES UMEN

Frente a los cambios en el mundo del trabajo contemporáneo, cuestiones como globalización, desreglamentación, (des)empleo, precarización, entre otras, hacen parte de las preocupaciones de investigadores y profesionales. En ese contexto emergen cuestionamientos sobre la centralidad del trabajo en sus dimensiones social y psicológica, así como los procesos de significación a él asociados. Este artículo presenta y analiza los principales abordajes teóricos identificados en las literaturas internacional y brasileña en el campo de la Psicología, que tratan de los significados y de los sentidos, en particular aquellos atribuidos al trabajo. Entre las diferentes bases epistemológicas que dan soporte a los estudios actuales fueron identificadas las siguientes: cognitivista, construccionista, sociohistórica, existencialista y de estudios culturales. Las conclusiones explicitan que los conceptos todavía carecen de mayor precisión, de modo que diferentes autores, muchas veces, adoptan significados y sentidos del trabajo como si ambos fueran el mismo fenómeno. Palabras clave autores

Trabajo, sentidos, significados, abordajes teóricos.

Palabras clave descriptores

Trabajo, teorías, psicología del trabajo, significación, (Psicología).

\section{A B S T R A C T}

Amidst changes in the contemporary world of labour, issues such as globalization, deregulation, (un)employment, precarization, among others, preoccupy researchers and professionals. In this context questions regarding the work centrality in its social and psychological dimensions emerge, as well as the meaning processes associated with working. This paper presents and analyzes the main theoretical approaches identified in the international and Brazilian literatures in the field of psychology making reference to meanings and senses, particularly those attributed to working. Different epistemological grounds supporting the current studies have been identified: cognitive, constructionist, cultural studies, existentialist and sociohistorical. The conclusions highlight that the concepts still lack precision. Different authors often adopt meanings and senses of working as if both were the same phenomenon.

Key words authors

Work, Senses, Meaning, Theoretical Approaches.

Key words plus

Work, Theory, Work-Psychological Aspects, Meaning (Psychology). 


\section{Introducción}

La sociedad contemporánea se caracteriza por una serie de transformaciones y paradojas que se hacen sentir en el trabajo, siendo éste una de las principales categorías de análisis social. Genéricamente, el trabajo remite a la capacidad del hombre de transformar la naturaleza y está asociado al alcance de objetivos.

La globalización, el proceso de reestructuración productiva, la competitividad, los cambios en los contratos de empleo, entre otros fenómenos, se han reflejado en la categoría trabajo. Emergen investigaciones dirigidas al estudio de la alienación, del desempleo, de la precarización de las relaciones de trabajo, de la crisis de las motivaciones. En medio de esas temáticas, es relevante, social y científicamente, comprender cuáles son los significados del trabajo en esta sociedad marcada por la multidiversidad y cuáles los sentidos personales engendrados por quienes viven de él.

Para tratar esta problemática, el presente artículo teórico incluye una breve revisión conceptual sobre el trabajo y sus transformaciones, y presenta diferentes concepciones sobre sus significados y sus sentidos. En el estudio de Tolfo, Coutinho, Baasch, Cugnier y Almeida (2005) fueron analizados diferentes estudios teóricos y empíricos sobre el tema. Se observó que en algunas investigaciones sobre los procesos de significación del trabajo son utilizadas indistintamente las palabras "significados" y "sentidos". En dicho artículo, mediante la revisión de la literatura, fueron identificadas diferentes bases teórico-epistemológicas que permiten definir los conceptos subyacentes a estos dos términos, relativos a cualquier proceso de significación o específicamente al trabajo. El presente estudio pretende hacer una contribución teórica en este campo de aplicación científica, a través de la exposición de esas diferentes perspectivas y de algunas aproximaciones entre ellas.

\section{El trabajo y sus transformaciones}

Los estudios sobre los sentidos y los significados del trabajo ganaron énfasis a causa de las transforma- ciones en las relaciones laborales y por el debate sobre la centralidad de la categoría trabajo (Antunes, 1998; Harvey, 2000; Offe, 1989).

El concepto de trabajo se está cambiando de acuerdo al contexto histórico y social (Blanch Ribas, 2003; Borges \& Yamamoto, 2004). Para Marx (1867/1968), el trabajo consiste en transformaciones con el objetivo de atender necesidades humanas. Se trata de una relación dialéctica entre hombre y naturaleza, en la cual la transformación de uno redunda en la transformación del otro, y viceversa. El trabajo también presupone la relación con otros hombres y puede ser comprendido como un esfuerzo colectivo, en el cual todos tendrían que participar.

De acuerdo con Oliveira, Piccinini, Fontoura y Schweig, "Es a través del trabajo que el ser humano ha buscado suplir sus necesidades, alcanzar sus objetivos y realizarse. Sin embargo, por su naturaleza amplia y compleja, influenciada por la cultura y momento histórico, este concepto puede modificarse" (2004, p. 334, traducción nuestra). En esa trayectoria, el trabajo asumió tanto connotaciones negativas como positivas (Bastos, Pinho, \& Costa, 1995). En las sociedades antiguas, tuvo origen una de las primeras concepciones, la clásica, en la cual el trabajo era concebido como tortura, tripalium, lo cual no valorizaba ni a la tarea ni al individuo.

$\mathrm{Al}$ abordar los valores y concepciones en relación con el trabajo, Blanch Ribas (2003) identifica tres posibilidades: polo negativo, centro del continuo y polo positivo. La primera está de acuerdo con la idea del tripalium, o sea, de castigo, coerción, esfuerzo y penalidad. En la posición central están las representaciones del trabajo en una perspectiva instrumental, en la medida en que él proporciona la supervivencia e implica dedicarse a la consecución de ese fin. El polo positivo del trabajo es asociado a posibilidades de satisfacción y de autorrealización, misión, valor. En la sociedad capitalista contemporánea, el trabajo está predominantemente asociado a valores positivos en la vida cotidiana, en razón de la valorización de la ética del trabajo influenciada por la doctrina protestante, conforme a lo argumentado por Weber (Blanch Ribas, 2003; Borges \& Yamamoto, 2004). 
De la misma forma, destacando las diferentes connotaciones que el trabajo puede asumir, Blanch Ribas (2003) propone una definición genérica, capaz de abarcar las especificidades de las situaciones concretas. Para el autor, el trabajo es una actividad humana social, compleja y dinámica, ejercida de forma individual o colectiva. No se reduce, las acciones instintivas resultantes de las funciones biológicas direccionadas a la supervivencia, sino que "(...) se distingue de cualquier otro tipo de práctica animal por su naturaleza reflexiva, consciente, propositiva, estratégica, instrumental y moral" (pp. 34-35).

En el siglo XX, hasta la década de 1970, el taylorismo/fordismo -caracterizado por la producción en serie y las empresas verticalizadas- predomina como forma de organización del trabajo y de la producción, y la centralidad de la categoría trabajo no se pone en cuestión. A partir de esa década, ese modelo de organización productiva da señales de agotamiento, por no mantener los índices de crecimiento del proceso de acumulación de capital, ni las tasas de empleo formal sostenidos hasta entonces. En el intento de retoma de patrones de acumulación anteriores, se identifican diferentes estrategias de reestructuración productiva, en especial aquellas asociadas a la llamada Tercera Revolución Industrial, con la introducción de la microelectrónica y la informatización de los procesos productivos. También se observa una tendencia a la substitución de las formas de organización productiva asociadas al taylorismo/fordismo, especialmente con la difusión del llamado modelo japonés (Antunes, 2000).

Con el capitalismo, el trabajo se asoció al sustento material y, en relación con el empleo, se caracterizó por relaciones contractuales asalariadas. Mientras el trabajo remite a la transformación de la naturaleza, el empleo está asociado, con mayor frecuencia, a relaciones contractuales, al vínculo formal con la organización, a las obligaciones y al salario (Borges \& Yamamoto, 2004). Con la reestructuración productiva, se verifica un aumento en la precarización de las relaciones y de las condiciones de trabajo, así como un significativo crecimiento de los índices de desempleo. Kovács (2005) identifica formas flexibles de empleo que emergen a partir de las últimas décadas del siglo XX (trabajo temporal, de tiempo parcial, a domicilio y autoempleo) en la sociedad capitalista contemporánea, como parte de la crisis del modelo de empleo predominante en los países de capitalismo avanzado.

La emergencia de relaciones diversificadas de trabajo, muchas veces, puede llevar al individuo a no identificarse con las actividades que realiza y a cuestionarse sobre sus significados y sentidos. Hay que cuestionar, también, las formas heterogéneas como esos cambios están establecidos en países económicamente más desarrollados y en aquellos considerados como periféricos. Conforme Pulido-Martínez (2007), la discusión de la centralidad del trabajo en parte de la "Psicología del primer mundo", implica la actuación de psicólogos para ayudar a las personas a responder de forma adecuada a los cambios e innovaciones implantadas para modernizar la sociedad. La adhesión al mainstream representa, según el mismo autor, "la destrucción violenta de la maneras tradicionales de vivir y pensar que constituyen las bases de la identidad" (p. 29).

El escenario de diversificación y de complejidad trae nuevos elementos para la relación hombre/ trabajo y analizarla implica considerar tanto las condiciones socioeconómicas en las cuales esa acción humana se desenvuelve, el significado, el sentido y el valor socioculturales de esa experiencia (Blanch Ribas, 2003). De la Garza Toledo (2008) enfatiza la necesidad de:

(...) un concepto ampliado de Trabajo, que considera a la vez sus dimensiones objetiva e subjetiva. Es decir, partir de que el trabajo es una forma de interacción entre hombres y con objetos materiales y simbólicos, que todo Trabajo implica construcción e intercambio de significados. (p. 1)

La diversidad de los "mundos del trabajo" genera, entre otras cosas, la pluralidad y el cuestionamiento

1 “(...) el concepto mundo del trabajo como separado de otros mundos de vida (familia, tiempo libre, estudio, etc.) es en parte una construcción social” (De la Garza Toledo, 2001, p. 29). 
sobre la centralidad de la categoría trabajo, tal como se refirió anteriormente, dado que éste estaría contribuyendo a la pauperización, la racionalización y la alienación, y estaría perdiendo principios éticos. De esa forma, "(...) su sentido para el operario no coincide con su significación objetiva” (Offe, 1989, p. 122, traducción nuestra).

El debate teórico sobre la crisis del trabajo puede asumir varias formas, pero, de acuerdo con De la Garza Toledo (2001, pp. 11-12), predomina la tesis de aquellos que afirman el fin del trabajo "como centro de la creación de identidad de sujetos sociales" y "como núcleo de la acumulación del capital" (asociado a la sobrevaloración del sector financiero y a la automatización de los procesos productivos), y describen las características de la crisis en la sociedad posindustrial y las formas que adquiere en América Latina. El autor inicia su análisis señalando el antiguo problema del concepto de trabajo como solamente restringido al sueldo. De este modo, la ampliación de formas flexibles de empleo, de acuerdo con Kovács (2005), significaría no el fin, sino el surgimiento de nuevas formas laborales, así como el vaciamiento de los lugares de poder ocupados por los trabajadores y por sus organizaciones. En el mismo sentido, De la Garza Toledo afirma:

La declinación del trabajo es sobre todo por la marginación de los trabajadores y sus organizaciones; es una crisis de deslegitimación. Su pérdida de centralidad no la ha substituido la simple fragmentación posmoderna sino otra centralidad: la del capital y la del empresario como sujeto. (p. 26)

Berger y Luckmann (2004) identifican, aun, la existencia de una crisis de sentidos en razón de la pluralidad de valores y de instituciones, que influencian los sujetos sociales en diferentes direcciones y perspectivas. Frente a esto, se considera relevante, social y científicamente, comprender los significados y los sentidos del trabajo en la sociedad contemporánea.

\section{Significados y sentidos del trabajo}

La temática de los significados y de los sentidos del trabajo ha sido investigada más sistemáticamente por los psicólogos, a partir de la década de 1970. Desde entonces, diferentes corrientes epistemológicas han venido dando soporte a la producción científica en este campo. Los investigadores brasileños Borges, Tamayo y Alves-Filho (2005) identificaron divergencias de pensamiento y de bases epistemológicas, entre los autores que estudian el significado del trabajo. Para ellos,

(...) parte de esas divergencias tienen origen en la interrelación entre la influencia del cognitivismo y la influencia de la visión de mundo de los investigadores (...) es posible diferenciar dos grupos (tipos) distintos, ambos vinculados a la influencia del cognitivismo (...). El primero de ellos (...), con influencia fenomenológica más fuerte, es designado como empírico-descriptivo. El segundo, en el cual se fortalece la influencia existencialista, es designado como grupo en transición. (p. 145, traducción nuestra)

Para ejemplificar las características de las investigaciones, identificadas por los autores como grupo empírico-descriptivo (fenomenológico), Borges et al. (2005) citan los estudios de Meaning of Working (MOW). Dicho grupo considera el fenómeno como multidimensional y constituido por tres variables: centralidad, normas dadas por la sociedad, resultados y objetivos valorados. ${ }^{2}$ En sus estudios se privilegian los instrumentos cuantitativos y se adopta el pensamiento sistémico, por medio de la combinación de variables, entre ellas: la ocupación de los individuos, la nacionalidad, el género, el grupo de edad y el nivel de instrucción. Las variables identificadas por investigadores del MOW han sido el fundamento de la mayoría de los estudios empíricos sobre el significado del trabajo y, algunas veces, también sobre el sentido, como veremos enseguida.

2 Los conceptos de las diferentes variables serán precisados posteriormente, cuando se trate la Producción del Grupo MOW. 
El grupo en transición (existencialista) considera que los componentes del significado del trabajo están en permanente proceso de construcción. El enfoque en el estudio de la estructura del significado del trabajo se centra en los nexos y contradicciones, lo que superaría la visión sistémica, en dirección a una visión dialéctica. Los significados del trabajo y/o sus componentes están asociados a las vivencias de los participantes de los estudios y combinan métodos cuantitativos y cualitativos (Borges et al., 2005).

En este artículo, identificamos como principales vertientes contemporáneas de la Psicología que tratan de significados y sentidos, ${ }^{3}$ las siguientes: cognitivista, existencialista, construccionista, de estudios culturales y sociohistórica. Se presentarán los principales presupuestos de cada una de esas perspectivas, iniciando por los conceptos y las definiciones adoptados por el Grupo MOW, dada su influencia en la mayoría de los estudios sobre el significado del trabajo.

El MOW tiene un programa de investigaciones sobre el tema, desde 1978. Según Ruiz-Quintanilla y Claes ${ }^{4}$ (2000), el significado del trabajo incluye valores, creencias, actitudes y expectativas, que las personas de la sociedad contemporánea han desarrollado en relación con el trabajo. Los significados son un componente de la realidad social, con implicaciones tanto en las acciones individuales como en los procesos grupales y en la sociedad.

Con base en estudios que privilegian la perspectiva cognitivista e investigaciones empíricas caracterizadas como investigación heurística y survey crossnational, los autores están desarrollando un programa de investigación para identificar semejanzas y diferencias del fenómeno, en diferentes países. Tras investigaciones longitudinales y comparativas, el MOW identificó tres variables que persistieron como constituyentes del fenómeno "significados del trabajo": centralidad, normas sociales, y valores asociados a los resultados e importancia de las metas de trabajo.

3 Algunos estudiosos están preocupados por conceptuar significados y sentidos de modo genérico, mientras que otros relacionan los conceptos al trabajo.

4 Ambos investigadores coordinaron el grupo MOW.
La centralidad está relacionada con la identificación personal con el trabajo y, para RuizQuintanilla y Claes (2000), "es definida como el grado de importancia que el trabajo tiene en la vida de un individuo en algún punto dado del tiempo" (p. 340, traducción nuestra). La centralidad está constituida por dos componentes: el valor atribuido al trabajo y su importancia en relación con otros papeles/funciones en la vida. Las normas sociales comprenden las creencias y expectativas sobre derechos y deberes relativos al trabajo. Los componentes normativos están asociados a dos tipos de normas: las relativas a la obligación, o sea, responsabilidad personal internalizada y compromiso organizacional (compromiso normativo); y las relativas a los derechos del trabajador (cambios justos en la relación entre empleados y empleadores, esfuerzos y resultados, inversiones y recompensas).

Los valores asociados a los resultados se refieren a las razones para trabajar, tales como estatus y prestigio, satisfacción, contactos sociales (RuizQuintanilla \& Claes, 2000). La importancia de las metas de trabajo está relacionada a estudios sobre satisfacción, valores e incentivos. Los investigadores del Grupo MOW identificaron dos dimensiones relacionadas con las metas individuales: aquellas relativas a aspectos económicos, instrumentales y extrínsecos (pago y seguridad en el empleo, por ejemplo) y las referidas a aspectos intrínsecos o expresivos, como autonomía y uso de habilidades.

En la perspectiva cognitivista, representada por la investigadora brasileña Lívia Borges (1997), el significado del trabajo es definido como una cognición multifacética, que tiene un carácter histórico, dinámico y subjetivo. Sus tres componentes principales son: a) una cognición subjetiva, que presenta una variación individual, reflejando la historia personal y muestra la forma como el individuo interpreta y da sentido al trabajo; b) una sociohistórica, que, además de presentar aspectos compartidos por un conjunto de individuos, refleja las condiciones históricas de la sociedad en la cual están insertados y c) una dinámica, como un 
constructo inacabado, en permanente proceso de construcción.

La cultura y la construcción de significados están entre aquellos aspectos básicos diferenciadores del ser humano como especie. Para Bruner (2000 citado en Borges, 1997), los significados son componentes mediadores de la relación del individuo con su mundo y causas de las acciones humanas, dotados de componentes afectivo-cognitivos elaborados por los individuos en la interrelación con la sociedad en la cual se insertan, constituyéndose como elementos de la cultura y, en consecuencia, como componentes fundadores de la propia condición humana (Bruner, 2000 citado en Borges \& Alves-Filho, 2003). Entonces, la construcción de significados es un proceso subjetivo que comprende tanto la historia del individuo, como su inserción social.

Para Borges (1998), los individuos construyen el significado del trabajo en su proceso de socialización, cuando se apropian de contenidos referentes a las concepciones formales del trabajo, a la estructura social de las organizaciones y a los aspectos socioeconómicos de las ocupaciones y del ramo de actividad. Borges $(1997,1999)$ argumenta aun que el individuo, al atribuir significados al trabajo o al resignificarlo, ora intenta justificar lo que ve, vivencia y oye el ambiente de trabajo, ora quiere explicaciones que hagan inteligibles sus vivencias, ora pretende crear un camino para sus acciones, ora puede tener otras posibilidadesLos estudios de Borges $(1997,1999)$ se basan en la producción teórico-empírica desarrollada por el grupo MOW. Ya en 1997, la autora adoptaba las siguientes variables: centralidad del trabajo, atributos descriptivos, atributos valorativos, y jerarquía y definición de los atributos. La centralidad relativa del trabajo presupone una jerarquización de las esferas de la vida (familia, trabajo, religión, ocio y comunidad). En sus investigaciones relativas al lugar ocupado por el trabajo entre las esferas de la vida, Borges (1997) verificó que este era precedido solamente por la familia, en grado de importancia. Los atributos descriptivos se refieren a la percepción del trabajo de como "es" concretamente y los atributos valorativos como "debe ser". Finalmente, la jerarquía de atributos remite a la organización escalonada de las características atribuidas al trabajo por los individuos.

Para Weick (1995), la creación de sentido es un proceso cognitivo que implica partir del cuestionamiento sobre la relación entre pensamiento y lenguaje y presenta siete propiedades: 1) tiene base en la construcción de la identidad, o sea, en el cuestionamiento sobre quién soy yo; 2) es retrospectiva, pues, para aprehender lo que pienso, retomo lo que dije antes; 3 ) presupone la interpretación de ambientes perceptibles; 4) es determinada socialmente, tanto por el proceso de socialización como por las expectativas sociales; 5) es continua, es decir está en curso; 6) tiene foco, o sea, solamente una pequeña parte del habla se destaca debido al contexto y a las disposiciones personales y 7) es regida más por la plausibilidad que por la exactitud.

En una perspectiva existencialista se encuentra Victor Frankl (2005), fundador de la llamada logoterapia $a^{5}$, para la cual "la búsqueda de sentido en la vida de la persona es la principal fuerza motivadora en el ser humano" (p. 92, traducción nuestra). El autor entiende el ser humano como una criatura responsable, que precisa realizar el sentido potencial de su vida, que no es interno a la persona, sino que es revelado en el mundo.

Adoptando una perspectiva que privilegia el existencialismo, con base en la obra de Victor Frankl y los estudios del Grupo MOW, se encuentran las investigaciones de Morin (2001), sobre sentidos del trabajo. Para esta autora, las principales razones que motivan las personas a trabajar son: actualizar sus potenciales; adquirir seguridad y ser autónomas; relacionarse con otros y tener sentimientos de pertenencia; prestar un servicio y hacer su contribución a la sociedad; y tener un sentido. El análisis de tales componentes permitió determinar tres conjuntos de características para que el trabajo tenga sentido: ser realizado en buenas condiciones; ofrecer oportunidades

5 La Tercera Escuela Vienesa de Psicoterapia es sinónimo de Logoterapia, cuyo término es formado por logos, que viene del griego y significa 'sentido'. 
para aprender y prestar servicios; ser interesante, variado y con mucha autonomía. De acuerdo con la autora el sentido es fuertemente influenciado por la propia organización del trabajo, pues ésta “(...) debe ofrecer a los trabajadores la posibilidad de realizar algo que tenga sentido, de practicar y desarrollar sus competencias, de desarrollar sus juicios y su libre albedrío, de conocer la evolución de sus desempeños y de ajustarse" (p. 9, traducción nuestra).

Basándose en los patrones de definición del trabajo determinados por England y Whiteley (1991 citados en Morin, 2001), la autora identifica seis categorías, cuyos patrones $\mathrm{A}, \mathrm{B}$ y $\mathrm{C}$ corresponden a concepciones positivas del trabajo: $\mathrm{A}$ ) actividad que agrega un valor a alguna cosa y por la cual hay que tener en cuenta de los resultados; B) actividad que proporciona a quien lo realiza un sentimiento de pertenencia y que conlleva una contribución a la sociedad; C) actividad que beneficia a otros, con contribución social y valor agregado. Los patrones D y E se refieren a los aspectos negativos: D) actividad que no es agradable, pero debe ser realizada por alguien en un lugar específico, bajo la supervisión de otra persona y E) actividad desagradable que comporta exigencias físicas y mentales. El patrón F corresponde a una concepción neutra del trabajo: actividad que sigue un horario regular en un local de trabajo y por el cual se recibe un salario.

Morin (1996, p. 269) comprende el sentido como "un efecto, un producto de la actividad humana". Se trata de una estructura afectiva constituida por tres componentes: la significación, la orientación y la coherencia. La significación presupone la aprehensión y comprensión de la experiencia fenoménica, así como el valor o la importancia atribuida a esa experiencia. La orientación es relativa al proyecto, la intención que dirige la acción del sujeto, una inclinación por un objeto, tomado en su sentido más amplio. La coherencia expresa la integración de las informaciones del campo de consciencia del sujeto, obtenidas a través de operaciones con el objetivo de equilibrar la vida interior y social.

En estudio realizado con jóvenes ejecutivos brasileños, Morin, Tonelli y Pliopas (2007) organi- zaron las características de un trabajo con sentido para quien lo ejecuta, en tres dimensiones: individual, organizacional y social. En la primera fue identificado como teniendo sentido aquel trabajo que permite satisfacción personal, independencia y supervivencia, crecimiento y aprendizaje, y es fuente de identidad. En su relación con la organización la persona da sentido a aquella actividad considerada como útil, que permite establecer relaciones e inserción social. Ya en la dimensión social fue valorizado el trabajo que contribuye a la sociedad. En esta concepción "las acciones de las personas precisan tener sentido", existe trabajo sin sentido y "el análisis de los sentidos del trabajo no es una cuestión unánime" (Morin et al., 2007, p. 49, traducción nuestra), lo que justifica la importancia de que se continúen los estudios sobre el fenómeno.

En el abordaje construccionista la realidad no existe independiente del modo en que las personas tienen que comprenderla, pues los sujetos y los objetos son entendidos como construcciones histórico-sociales. Por medio de la socialización primaria, el sujeto es puesto en contacto con el mundo, con sus reglas e instituciones, mediadas por los otros significativos, construyendo, así, su identidad. Desde temprana edad, los padres -o sus sustitutos- enseñan en qué consiste la realidad predominante en la vida cotidiana: normas, reglas, tabúes, roles, etc. (Goffman, 1985). La identidad va construyéndose, también, con la socialización secundaria, en la cual la interacción con los otros proporciona datos que, generalmente, corroboran los aprendizajes iniciales. Con la intersubjetividad, el individuo permanece en aprendizaje constante sobre su universo, de modo que aquello que él vivencia o le es enseñado en la vida cotidiana pasa a ser incorporado como "(...) la realidad. No requiere mayor explicación que se extienda además de su simple presencia. Está simplemente ahí... Sé que es real" (Berger \& Luckmann, 1985, pp. 40-41, traducción nuestra). Entonces, la comprensión de la realidad se construye sobre la base del sentido común, y es con fundamento en esos conocimientos prácticos como las personas constituyen sus significados (Spink, 2004). Autores que adoptan 
el abordaje construccionista afirman la indisolubilidad entre las significaciones producidas por los sujetos y la realidad (Berger \& Luckmann, 2004; Spink, 2004).

En el campo de la Psicología Social brasileña se ha rescatado el abordaje sociológico de Berger y Luckmann, particularmente haciendo énfasis en "(...) enfocar justamente el momento de la interacción, o sea, los procesos de producción de sentidos en la vida cotidiana" (Spink, 2004, p. 26). Es a través de los conocimientos adquiridos del sentido común, producidos cotidianamente, como las personas dan sentido al mundo del cual hacen parte. En esta perspectiva, el sentido es concebido como:

(...) una construcción social, un emprendimiento colectivo más precisamente interactivo, por medio del cual las personas, en la dinámica de las relaciones sociales, históricamente datadas y culturalmente ubicadas, construyen los términos a partir de los cuales comprenden y lidian con las situaciones y fenómenos a su alrededor (Spink \& Medrado, 2004, p. 41, traducción nuestra).

El fundamento de la constitución de sentido está en las vivencias subjetivas. La constitución subjetiva del sentido es el origen de todo el acervo social del conocimiento. Las experiencias almacenadas socialmente (historia e instituciones) ayudan al individuo en la solución de problemas ya identificados anteriormente. "En general, la parte accesible del reservorio del sentido constituye el cierne del entendimiento común, con cuyo auxilio el individuo tiene que arreglárselas en el medio ambiente natural y social de su época" (Berger \& Luckmann, 2004, p. 20, traducción nuestra). El conocimiento desarrollado por medio del sentido común es importante para los construccionistas sociales, pues tiene en consideración el entendimiento que las personas tienen de la realidad diaria en la cual se desarrollan. La producción de los sentidos en la cotidianidad ocurre por medio de las prácticas discursivas, que "implican acciones, selecciones, escogencias, lenguajes, contextos, en fin, una variedad de producciones sociales de las cuales son expresión” (Spink \& Frezza, 2004, p. 38, traducción nuestra).

La perspectiva construccionista se articula, algunas veces, con los Estudios Culturales. Guareschi, Medeiros y Bruschi (2003) son autores brasileños que establecen tal articulación. Para ellos, el construccionismo social:

(...) tiene la importancia de una epistemología que tiene la formación discursiva y el proceso de significación como principales presupuestos; propone la constatación de que la representación es del orden de los sentidos que el sujeto atribuye, tanto en su ámbito consciente como no consciente, a su contexto social y cultural. De esa forma, se busca inquirir qué dicen, piensan y actúan los sujetos, acerca de los sentidos que constituyen el mundo y, de modos diversos, el propio sujeto (...) (Guareschi, Oliveira, Giannechini, Comunello, Nardini \& Pacheco, 2003, p. 47, traducción nuestra).

Guareschi, Medeiros y Bruschi (2003) retoman investigadores de la cultura, en particular a Stuart Hall, para destacar el foco en el estudio de fenómenos culturales en la sociedad contemporánea, comprendidos en su propio contexto y críticos de una perspectiva etnocéntrica. Coherentes con la perspectiva de multidisciplinaridad, los estudiosos adoptan teorías diversas, sin apegarse a un marco epistemológico específico, incluyendo el marxismo, el psicoanálisis, el posestructuralismo y el posmodernismo (Nelson, Treichler \& Grossberg, 2003).

Para esos estudios, la cultura es el centro y se define con base en los contextos político, económico, histórico y social, de los cuales surge y con los cuales se construye. Entendida como práctica de significación, la cultura adquiere un papel constitutivo y activo, no dependiente del análisis social. Considerada la relación entre historia y cultura, ésta última contempla el espacio de disputas y reproducciones, en el cual se generan los sentidos, y los sujetos que componen los diversos grupos sociales.

A través de las prácticas culturales, las personas son llevadas a actuar y a socializarse de ciertos modos, en medio de una verdadera disputa entre la 
cultura, para definir determinados sentidos, y las manifestaciones o resistencias por parte de las personas (Guareschi, Oliveira et al., 2003). La cultura y el lenguaje están íntimamente vinculados, pues éste es el mecanismo esencial por medio del cual la cultura produce significados sociales. El concepto de lenguaje pasa a tener un nuevo dimensionamiento, con un lugar especial en la construcción de los sentidos. Al nombrar los objetos, los sujetos crean el mundo en el cual actúan, pues, a través de los sistemas de significación, hacemos el mundo inteligible, operacional y constituido de sentidos (Bernardes \& Hoenisch, 2003).

Lo que las personas consideran conceptos "naturales" hace parte de fenómenos discursivos a través de los cuales se produce no solo la realidad, sino también los sujetos (Guareschi, Medeiros \& Bruschi, 2003). La identidad de éstos es, entonces, entendida como una "posición de sujeto", sobre cómo las personas se vuelven alguien en determinado grupo social; por ejemplo, en la etnia, en el género, en la profesión o en la familia. De este modo, el concepto de identidad deja de ser entendido como esencia y pasa a ser comprendido como plural e inestable; las construcciones históricas y culturales se tejen permanentemente en redes discursivas. La "posición de sujeto" es una condición en las redes discursivas culturales como puntos de vista en una estructura social que siempre está por hacerse (Guareschi, Medeiros \& Bruschi, 2003). De acuerdo con Bernardes y Hoenisch (2003) el sujeto concebido por el posestructuralismo es direccionado a "hacerse", pues en lugar de generar prácticas sociales, el sujeto es construido por esas prácticas.

La contribución de la perspectiva sociohistórica también fue identificada, ya que, en ese abordaje, de acuerdo con Leontiev (1978), la significación es una generalización de las prácticas sociales de la realidad, correspondiendo a las representaciones y conocimientos en una época y una sociedad dadas, fijadas principalmente por medio del lenguaje. El autor atribuyó un doble sentido al término significación: "(...) en ciertos casos él designa significación de una palabra (significación verbal), en otros, los conocimientos, el contenido de la consciencia social asimilada por el individuo" (p. 130, traducción nuestra). Las transformaciones en las condiciones de existencia de la vida humana tienen, en esta perspectiva, profundas implicaciones en la estructura psicológica de los sujetos. De este modo, la principal modificación en la producción de la consciencia -con el desarrollo de la sociedad de clases- está relacionada con los cambios entre el plano de los sentidos y el plano de las significaciones.

Un aporte teórico importante para el análisis de los sentidos y los significados en la perspectiva sociohistórica, fue desarrollado por otro de sus precursores, el ruso Vygotski (1991), también fundamentado epistemológicamente en los principios del materialismo histórico-dialéctico. Al considerar al hombre como un ser eminentemente social reafirma la relación inexorable entre sujeto y sociedad. El desarrollo de la cultura marca el momento de ruptura en la historia humana, en el que el hombre pasa a transformar la naturaleza por el trabajo, creando sus propias condiciones de existencia y, de esa forma, recreándose a sí mismo en una doble y simultánea transformación.

El trabajo, como proceso de doble transformación, es posible gracias a la utilización de instrumentos mediadores, creados por los hombres, los cuales pueden ser de dos especies: físicos, producidos para actuar sobre la naturaleza, o semióticos (signos), producidos para posibilitar la comunicación y la representación de la realidad (Pino, 2000). En su obra Vygotski da relieve especial a la mediación semiótica ${ }^{6}$, comprendiendo que ésta tiene el papel de estructurar la consciencia humana (1991 citado en Pino, 1995). Gracias al sistema de signos, especialmente el lingüístico, el hombre puede nombrar y pensar los objetos y sus experiencias, y también compartirlos con los demás. En ese proceso, la actividad humana ocurre en doble dirección, ya que el sujeto al transformar la realidad se objetiva y, transformándose a sí mismo, se subjetiva.

6 La función semiótica equivale al desdoblamiento de lo real en su forma simbólica (Pino, 1995). 
La significación ocurre en las interacciones sociales y dice respeto al proceso de producción de sentidos y a los significados por ellos vehiculados, aquello que el signo significa para los sujetos. El significado es un componente indispensable de la palabra; es generalizable, por tanto, compartido socialmente; se caracteriza en la zona más estable del sentido, pero, entre tanto, no es inmutable. Hay, según Vygostki (1991), una preponderancia del sentido sobre el significado, y a cada palabra se le pueden atribuir diversos significados, los cuales serán transformados en sentidos singulares, de acuerdo con las emociones y necesidades que motivaran su uso. De este modo, el sentido de la palabra es, para el autor, la suma de todos los eventos psicológicos evocados en nuestra consciencia por medio de la palabra.

La definición de sentido en la obra de Vygotski (1991) no se restringe solamente a la palabra como unidad fonética, sino más bien la relaciona con su contexto. La evocación de los eventos psicológicos también se extiende más allá de la palabra, comprendiendo los signos como un todo.

(...) nunca abarcamos el sentido completo de la cosa y tampoco el sentido completo de las palabras. La palabra es una fuente inagotable de nuevos problemas, y su sentido nunca está acabado. El sentido de las palabras depende conjuntamente de la interpretación del mundo de cada uno y de la estructura interna de la personalidad. (p. 334, traducción nuestra)

El sentido dice respeto a la actividad del sujeto, en su relación con la vida y a la forma como los fenómenos objetivos son aprehendidos por su consciencia. El sentido personal y subjetivo de determinada significación depende de su apropiación o no, así como del grado y forma como es asimilada por el sujeto, individualmente, y el significado remite a la construcción colectiva (Basso, 1998).

Aguiar (2006) también identifica la construcción de los significados como sociales y los sentidos como relativos a la subjetividad. La autora explica que las categorías sentido y significado, pese a ser diferentes, no pueden ser comprendidas de manera desvinculada una de la otra y afirma:

\begin{abstract}
Los significados son, pues, producciones históricas y sociales. Son ellos los que permiten la comunicación, la socialización de nuestras experiencias. A pesar de ser más estables, "diccionarizados", ellos también se transforman en el movimiento histórico, momento en que su naturaleza interna se modifica, alterando, consecuentemente, la relación que mantienen con el pensamiento, entendido como un proceso. Los significados se refieren, así, a los contenidos instituidos, más fijos, compartidos, que son apropiados por los sujetos, configurados a partir de sus propias subjetividades. (p. 14, traducción nuestra)
\end{abstract}

La misma autora considera el sentido como "mucho más amplio que el significado, pues el primero constituye la articulación de los eventos psicológicos que el sujeto produce ante la realidad" (Aguiar, 2006, p. 14, traducción nuestra). El sujeto asume, de esta manera, la condición de autor al poder atribuir diversos sentidos a lo socialmente establecido, al mantener, como dice Zanella (2004), una relación marcada por movimientos de aceptación, oposición, confrontación, indiferencia, produciendo siempre nuevas e infinitas posibilidades de significación de la cultura y de las actividades sociales como un todo.

\section{Posibles aproximaciones entre los abordajes}

En este texto fueron estudiadas las siguientes perspectivas teórico epistemológicas: cognitivista, existencialista, construccionista, de estudios culturales, y sociohistórica. La diversidad de abordajes es representativa del modo como la Psicología se constituye como campo de conocimiento compuesto por diferentes escuelas psicológicas que definen los fenómenos de acuerdo a sus concepciones de hombre y sociedad. Estos abordajes han sido empleados en estudios sobre el trabajo y, usualmente, se observa la predominancia de la utilización de una perspectiva teórica. Como ejemplo se pueden citar Bastos, Pinho y Costa (1995) y Borges et al. (2005) que adoptan la perspectiva cognitivista, mientras Morin (2001) y Morin et al. (2007) asumen el referente existencialista, a 
pesar de que todos hagan referencia a los estudios del grupo MOW.

El propósito de este artículo fue justamente marcar las peculiaridades en las comprensiones de cada referente teórico sobre los procesos de significación. Entre tanto, es posible identificar convergencias entre las perspectivas presentadas.

Un aspecto que permite identificar puntos de convergencia entre las diferentes perspectivas se refiere al papel de la cultura en la producción de los sentidos y significados. En todos los estudios analizados, la cultura y la sociedad son elementos fundamentales para la comprensión de las significaciones. Es por medio de la cultura y de los aprendizajes sociales que el sujeto se construye y es construido. Aún así, los estudios culturales se diferencian de los demás -generalmente vinculados a filiaciones teóricas más unificadas-por explicitar diversas vinculaciones: marxismo, psicoanálisis, posestructuralismo y posmodernismo.

La importancia de la cultura, referida al lenguaje y la construcción de las significaciones, es una preocupación común a los investigadores cuyo fundamento está en los estudios culturales, construccionistas y socio-históricos. Esa articulación se justifica por el hecho de que, para autores como Bernardes y Hoenisch (2003), Spink (2003) y Leontiev (1978), por ejemplo, es por medio del lenguaje que la cultura vehicula los significados sociales. Es un mecanismo esencial para la fijación de la significación, que constituye una generalización de prácticas sociales de la realidad, de acuerdo con las representaciones de la época y de la sociedad (Leontiev, 1978).

Al tratar de los procesos de significación, algunos autores utilizan solamente la palabra "significados" (Borges, 1997, 1999; Borges et al., 2005; Ruiz-Quintanilla \& Claes, 2000); otros prefieren usar "sentidos" (Guareschi, Medeiros \& Bruschi, 2003; Morin, 2001; Morin et al., 2007; Spink, 2004); por último, hay aquellos que adoptan una diferenciación entre los dos conceptos (Aguiar, 2006; Basso, 1998; Berger \& Luckmann, 2004; Vygotski, 1991). Igualmente las diferencias, en algunos casos, pueden ser atribuidas a problemas lin- güísticos o de traducción, por lo cual identificamos ahí la necesidad de mayor precisión conceptual.

Los diferentes abordajes demuestran que, aunque la temática esté tomando cada vez más auge, es compleja y aún está en construcción. Algunos autores utilizan definiciones de significados y de sentidos del trabajo como si estuvieran tratando el mismo fenómeno y en un mismo nivel de análisis, lo que dificulta la construcción de un conocimiento más amplio sobre la cuestión.

\section{Consideraciones finales}

El trabajo en el contexto contemporáneo asume formas plurales. En la perspectiva marxista, que predomina en la literatura, el trabajo puede ser comprendido de forma genérica, como una transformación de la naturaleza para atender las necesidades humanas. Por su lado, el empleo configura una forma de trabajo asalariado, tradicionalmente característica del capitalismo, lo que ha generado una equiparación equivocada de los significados relativos a los dos conceptos. Frente la variedad de formas de empleo vigentes en la actualidad, se suman la diversidad, la heterogeneidad y la complejidad de la clase trabajadora, incluyendo a "(...) todos aquellos y aquellas que venden su fuerza de trabajo a cambio de salario (...)" (Antunes, 2000, p. 103, traducción nuestra), lo mismo que en relaciones precarias, de subcontratación, informales, temporales, etc. El carácter polisémico del trabajo presupone, también, considerar la necesidad de ampliar los conceptos existentes y tener en consideración sus aspectos tanto subjetivos como objetivos (Blanch Ribas, 2003; De La Garza Toledo, 2008).

La conceptualización teórica del trabajo se ve afectada por las visiones de las diversas áreas o ciencias dedicadas a su estudio. Esto implica pensar en la apropiación del concepto de centralidad utilizado en una perspectiva sociológica -clásica- que identifica el trabajo como principal hecho social y categoría sociológica clave. La centralidad del trabajo también se analiza desde una perspectiva psicológica, o sea, como la identificación de cuán 
central y, por tanto, pleno de significado para las personas, es el trabajo. Este debate sobre la indivisibilidad de las construcciones colectivas (sociales) y las singulares (individuales) relativas al trabajo, es fundamental para el avance de los estudios en el área, de la misma forma que, conceptualmente, se pueden definir objetos específicos de cada ciencia. Intersecciones y aproximaciones como estas remiten a reflexionar, también, sobre la creciente diversificación de los significados colectivos atribuidos al trabajo y al empleo, así como de los sentidos personales aprendidos en cotidianota cotidianidad de las diferentes formas de trabajo.

Se reiteran las conclusiones de Tolfo y Piccinini (2007), que afirmaron la importancia de estudios de atribución de los significados y de los sentidos del trabajo, en una perspectiva multidisciplinar, por abordar aspectos psicosociales multidimensionales y dinámicos, resultantes de la interacción entre elementos personales y ambientales. Por otro lado, la dificultad en establecer un "paradigma" en relación a lo que significa trabajar y lo que es una actividad laboral para quién la ejecuta, puede estar asociada a la diversidad, a la modernidad y al pluralismo, conforme aseveran Berger y Luckmann (2004). Para esos autores, la indivisibilidad de los sentidos es posible cuando existen comunidades de vida que tienen como función mantener el sentido, o sea, grupos sociales que comparten experiencias semejantes en la vida cotidiana y son portadores de valores sociales. Las comunidades, al compartir las experiencias de vida, representan la acción que se repite con regularidad, lo que es verificable en relaciones sociales más durables. Las instituciones tienen un papel importante para la permanencia y durabilidad de la comunidad, que presupone que se comparta un mínimo de valores y de sentido. En la medida en que los científicos, actualmente, también están sujetos a la crisis de las instituciones y al zeitgest, suelen encontrar más dificultades para mantener regularidad en los significados y en los sentidos del trabajo y, consecuentemente, en su conceptualización.

La comprensión de la cultura articulada a la acción se encuentra en autores de todas las pers- pectivas estudiadas. Hay unanimidad en el entendimiento de que el sentido de la acción del sujeto no es pautado solamente por factores intrínsecos, sino construido socialmente, en el ámbito de la cultura. A pesar de las diferencias, los abordajes sobre sentidos y significados presentados, tienen en común la concepción que éstos son producidos por los sujetos a partir de sus experiencias concretas en la realidad. Por esta razón, en este estudio proponemos comprender los significados como construcciones elaboradas colectivamente, en determinado contexto histórico, económico y social concreto. Si ya los sentidos consisten en una producción personal dependiente de la aprehensión individual de los significados colectivos, en las experiencias cotidianas, es importante, entonces, resaltar las transformaciones por las que pasan los sentidos y los significados, ya que son construidos en una relación dialéctica con la realidad.

Los significados y los sentidos del trabajo han sido objeto de estudio más reciente, especialmente en el curso de la llamada crisis del trabajo, como apunta De la Garza Toledo (2001), y otros autores del campo de la Sociología. Los diferentes abordajes psicológicos sobre significados y sentidos del trabajo presentados en este texto, corresponden a las concepciones teóricas predominantes en esta ciencia. Si, de un lado, existen diversos estudios que enfatizan la centralidad del trabajo y la predominancia de concepciones positivas, la Psicología necesita ser capaz de entender mejor las contradicciones, las disyunciones, que hacen que el trabajo pueda ser fuente de placer, y también de sufrimiento (Cimbalista, 2007). En esta dirección el diálogo con teorías críticas, como el construccionismo y el abordaje socio-histórico, se puede constituir en un fundamento consistente para estudios sobre los procesos de significación en el trabajo. Más que optar por una determinada teoría, se defiende la posición de comprender los sujetos trabajadores insertados en un determinado contexto político, económico y social cuya racionalidad, autonomía, eficacia y participación dependen de la realidad social allí construida. 


\section{Referencias}

Aguiar, W. M. J. (2006). A pesquisa junto a professores: fundamentos teóricos e metodológicos. En W. M. J. Aguiar (Org.), Sentidos e significados do professor na perspectiva sócio-histórica: relatos de pesquisa (pp.1122). São Paulo: Casa do Psicólogo.

Antunes, R. (1998). Adeus ao trabalho? Ensaio sobre as metamorfoses e a centralidade do mundo do trabalho (5a ed.). São Paulo: Cortez.

Antunes, R. (2000). Os sentidos do trabalho: ensaio sobre a afirmação e a negação do trabalho (2a ed.). São Paulo: Boitempo.

Basso, I. S. (1998). Significado e sentido do trabalho docente. Cadernos CEDES, 19 (44), 19-32. Recuperado el 14 diciembre, 2003, de http://www.scielo. br/scielo.php?script $=$ sci_arttext $\&$ pid $=$ S0101 . $2621998000100003 \& \operatorname{lng}=\mathrm{pt} \& n r m=$ iso

Bastos, A. V. B., Pinho, A. P. \& Costa, C. A. (1995). Significado do trabalho: um estudo entre trabalhadores de organizações formais. Revista de Administração de Empresas, 35 (6), 20-29.

Berger, P. L. \& Luckmann, T. (1985). A construção social da realidade (11a ed.). Petrópolis: Vozes.

Berger, P. L. \& Luckmann, T. (2004). Modernidade, pluralismo e crise do sentido - A orientação do homem moderno. Petrópolis: Vozes.

Bernardes, A. G. \& Hoenisch, J. C. D. (2003) . Subjetividade e Identidades: possibilidades de interlocução da Psicologia Social com os Estudos Culturais. En M. F. Guareschi \& M. E. Bruschi (Orgs.), Psicologia Social nos estudos culturais: perspectivas e desafios para uma nova psicologia social (pp. 95-126). Petrópolis: Vozes.

Blanch Ribas, J. M. (2003). Trabajar en la modernidad industrial. En J. M. Blanch Ribas, M. J. E. Tomás, C. G. Durán \& A. M. Artiles (Eds.), Teoría de las relaciones laborales. Fundamentos (pp. 19-147). Barcelona: Editorial UOC.

Borges, L. O. (1997). Os atributos e a medida do significado do trabalho. Psicologia: Teoria e Pesquisa, 13(2), 211-221.

Borges, L. O. (1998). Os pressupostos dos estudos do significado do trabalho na psicologia social: no caminho do existencialismo. Vivência, 12 (2), 87-105.
Borges, L. O. (1999). As concepções do trabalho: um estudo de análise de conteúdo de dois periódicos de circulação nacional. Revista de Administração Contemporânea, 3(3), 81-108.

Borges, L. O., Tamayo, A. \& Alves Filho, A. (2005). Significado do trabalho entre profissionais da saúde. En L. O. Borges (Org.), Os profissionais de saúde e seu trabalho (pp. 143-197). São Paulo: Casa do Psicólogo.

Borges, L. O. \& Yamamoto, O. H. (2004). O mundo do trabalho. En J. C. Zanelli, J. E. Borges-Andrade \& A. V. Bastos (Eds.), Psicologia, organizações e trabalho no Brasil (pp. 25-62). Porto Alegre: Artmed.

Borges, L. O. \& Alves-Filho, A. (2003). Manual de aplicação do IMST. Inventário da Motivação e Significado do Trabalho. Manuscrito no publicado.

Cimbalista, S. (2007). Sufrimiento: los significados adversos de las condiciones de trabajo en el sistema de producción flexible. Universitas Psychologica, 6(1), 69-78.

De la Garza Toledo, E. (en prensa). Hacia un concepto ampliado de trabajo. En E. De la Garza Toledo (Ed.), Trabajo, calificación e identidad. Recuperado el 30 noviembre, 2009, de http://docencia.izt.uam. $\mathrm{mx} /$ egt/publicaciones/capituloslibros/Haciaunconceptoampliado.pdf

De la Garza Toledo, E. (2001). Problemas clásicos y actuales de la crisis del trabajo. En E. De la Garza Toledo \& J. C. Neffa (Comps.), El Futuro del Trabajo. El Trabajo del futuro (pp. 11-31). Buenos Aires: CLACSO.

Frankl, V.E. (2005). Um sentido para a vida. Aparecida: Idéias e Letras.

Goffman, E. (1985). Estigma: notas sobre manipulação da identidade deteriorada. Rio de Janeiro: Zahar.

Guareschi, N., Medeiros, P. \& Bruschi, M. (2003). Psicologia social e estudos culturais: rompendo fronteiras na produção do conhecimento. En N. Guareschi \& M. Bruschi (Orgs.), Psicologia social nos estudos culturais: perspectivas e desafios para uma nova psicologia social (pp. 23-49). Petrópolis: Vozes.

Guareschi, N. M. F., Oliveira, F. P., Giannechini, L. G., Comunello, L. N., Nardini, M. \& Pacheco, M. L. (2003). Pobreza, violência e trabalho: a produção de sentidos de meninos e meninas de uma favela. Estudos de Psicologia, 8(1), 45-53. Recuperado 
en 20 de noviembre. 2009, de http://www.scielo. br/scielo.php?script $=$ sci_arttext\&pid $=$ S1413 . 294X2003000100006\&tlng $=$ en\&lng $=$ en\&nr $\mathrm{m}=$ iso.

Harvey, D. (2000). Condição pós-moderna: uma pesquisa sobre as origens da mudança cultura (9a ed.). São Paulo: Loyola.

Kovács, I. (2005). Emprego flexível em Portugal: alguns resultados de um projecto de investigação. En I. Kovács (Org.), Flexibilidade no emprego: riscos e oportunidades (pp. 11-53). Oeiras, Portugal: Celta.

Leontiev, A. (1978). O desenvolvimento do psiquismo. Lisboa: Livros Horizonte.

Marx, K. (1968). Processo de trabalho e processo de produzir mais-valia. En K. Marx. O Capital (Vol. 1, pp. 201-223). Rio de Janeiro: Civilização Brasileira. (Originalmente publicado en 1867).

Morin, E. M. (1996). L'efficacité organisationnelle et sens du travail. En T.C. Pauchant et al. (Coords.), La quéte du sens. Gérer nos organisations pour la santé des personnes, de nos sociétés et de la nature (pp. 257-286). Montréal, Québec et Paris: Éditions d'Organisation.

Morin, E. M. (2001). Os sentidos do trabalho. Revista de Administração de Empresas, 41 (3), 8-19.

Morin, E., Tonelli, M. J. \& Pliopas, A. (2007). O trabalho e seus sentidos [Ejemplar especial 1]. Psicologia e Sociedade, 19, 47-56.

Nelson, C., Treichler, P. \& Grossberg, L. (2003). Estudos culturais: uma introdução. En T. Silva (Org.), Alienígenas na sala de aula: uma introdução aos estudos culturais em educação (5a ed., pp. 7-38). Petrópolis: Vozes.

Offe, C. (1989). Trabalho: a categoria sociológica chave? In C. Offe (Ed.), Capitalismo desorganizado (pp. 167-198). São Paulo: Brasiliense.

Oliveira, S. R., Piccinini, V. C., Fontoura, D. S. \& Schweig, C. (2004). Buscando o sentido do trabalho [CD-ROM]. En Anais do XXVIII Encontro da Associação Nacional de Pós-Graduação e Pesquisa em Administração, Porto Alegre, RS: ANPAD.

Pino, A. (1995). Semiótica e cognição na perspectiva histórico-cultural. Temas em Psicologia, 2, 31-39.
Pino, A. (2000). O social e o cultural na obra de Vigotski. Educação $\mathcal{B}$ Sociedade, 71, 45-79.

Pulido-Martínez, H. C. (2007). Produciendo trabajadores modernos: conocimiento psicológico y el mundo del trabajo en el sur. Universitas Psychologica, 6(1), 27-37.

Ruiz-Quintanilla, S. A. \& Claes, R. (2000). MOW Research Programs. En J. A. Katz (Ed.), Databases for the study of entrepreneurship (pp. 335-391). New York: JAI/Elsevier Science Inc.

Spink, M. J. (2004). Linguagem e produção de sentidos no cotidiano. Porto Alegre: EDIPUCRS.

Spink, M. J. \& Frezza, R. M. (2004). Práticas discursivas e produção de sentidos: a perspectiva da psicologia social. En M. J. Spink (Ed.), Práticas discursivas e produção de sentidos no cotidiano: aproximações teóricas e metodológicas (3a ed., pp. 17-39). São Paulo: Cortez.

Spink, M. J. \& Medrado, B. (2004). Produção de sentidos no cotidiano: uma abordagem teórico-metodológica para análise das práticas discursivas. En M. J. Spink, Práticas discursivas e produção de sentidos no cotidiano: aproximações teóricas e metodológicas (3a ed., pp. 41-63). São Paulo: Cortez.

Tolfo, S. R., Coutinho, M. C., Baasch, D., Cugnier, J. S. \& Almeida, A. R. (2005). Revisitando abordagens sobre sentidos e significados do trabalho [CD-ROM]. En Anais do II Fórum CRITEOS, Porto Alegre.

Tolfo, S. da R. \& Piccinini, V. (2007). Sentidos e significados do trabalho: explorando conceitos, variáveis e estudos empíricos brasileiros [Edición Especial 1]. Psicologia e Sociedade, 19, 38-46.

Vygotski, L. S. (1991). Obras escogidas II: problemas de psicología general. Madrid: Visor Distribuciones.

Weick, K. E. (1995). Sensemaking in organizations. Thousand Oaks: Sage.

Zanella, A. V. (2004). Atividade, significação e constituição do sujeito: considerações à luz da psicologia histórico-cultural. Psicologia em Estudo, 9(1), 127-135. 